MATHEMATICS OF COMPUTATION

Volume 68, Number 226, April 1999, Pages 733-747

S 0025-5718(99)01080-7

\title{
ERROR ESTIMATES FOR SCATTERED DATA INTERPOLATION ON SPHERES
}

\author{
KURT JETTER, JOACHIM STÖCKLER, AND JOSEPH D. WARD
}

\begin{abstract}
We study Sobolev type estimates for the approximation order resulting from using strictly positive definite kernels to do interpolation on the $n$-sphere. The interpolation knots are scattered. Our approach partly follows the general theory of Golomb and Weinberger and related estimates. These error estimates are then based on series expansions of smooth functions in terms of spherical harmonics. The Markov inequality for spherical harmonics is essential to our analysis and is used in order to find lower bounds for certain sampling operators on spaces of spherical harmonics.
\end{abstract}

\section{INTRODUCTION}

The purpose of this paper is to study Sobolev-type estimates for the approximation order resulting from using strictly positive definite kernels to do interpolation on the unit sphere $S^{n-1} \subset \mathbb{R}^{n}, n \geq 2$. In this paper, estimates on the rate of convergence of interpolants are obtained when the nodes for the interpolation are in arbitrary position. Denoting the set of such nodes by $X \subset S^{n-1}$, we call

$$
h(X):=\sup _{\mathbf{p} \in S^{n-1}} \inf _{x \in X} d(\mathbf{p}, x)
$$

the mesh norm of $X$, where $d$ is the spherical distance on the unit sphere, hence

$$
d(\mathbf{p}, \mathbf{q})=\arccos (\mathbf{p} \cdot \mathbf{q}), \quad \mathbf{p}, \mathbf{q} \in S^{n-1} .
$$

We study the interpolation problem in an appropriate Hilbert space $\mathbf{H}$ of continuous functions on $S^{n-1}$, which is defined by an underlying positive definite kernel $\kappa$. This space is often called the 'native space' associated with the kernel $\kappa$ in the literature on radial basis functions. Our aim is to establish uniform error estimates for the interpolant $u_{f}=u_{f, X, \kappa}$, which is of the form

$$
u_{f}(\mathbf{p})=\sum_{x \in X} c_{x} \kappa(x, \mathbf{p}) \quad \text { with } \quad c_{x} \in \mathbb{C},
$$

and which agrees with $f$ in the nodes $X$. Employing the associated norm of the Hilbert space, our estimates take the form

$$
\left\|f-u_{f}\right\|_{\infty} \leq \mathrm{const} \cdot h^{\alpha}\|f\|_{\mathbf{H}}, \quad f \in \mathbf{H} .
$$

Received by the editor August 25, 1997.

1991 Mathematics Subject Classification. Primary 41A05, 41A25; Secondary 41A30, 41A63.

Key words and phrases. Scattered data interpolation, spherical harmonics, Markov inequality, norming set, best approximation.

Research supported by NSF Grant DMS-9303705 and Air Force AFOSR Grant F49620-95-10194 .

(C)1999 American Mathematical Society 
The rate $\alpha$ in (2) is related to the smoothness of the kernel $\kappa$ and the smoothness of the function $f$ to be interpolated. Thus, these results may be seen as a successful extension to $S^{n-1}$ of related work of Duchon [5], and Madych and Nelson [12].

There has been an increasing awareness of the importance of approximation on the sphere with obvious applications to meteorology, oceanography and satellitebased techniques such as the Global Positioning System (GPS). For a nice account of other potential applications, see [8].

Over the last several years, there has been much fundamental work done by Freeden and colleagues [7] as well as by Wahba [21], [22] concerning approximation on $S^{n-1}$. Nevertheless, even though spherical positive definite functions, as introduced by Schoenberg [19], have been around for years, cf. Cheney [2], the approximation power of such functions on $S^{n-1}$ has not been nearly as well understood as on $\mathbb{R}^{s}$. For instance, the previously known rates of approximation of a given function $f$ by interpolants had been $\mathbf{O}(h)$ regardless of any underlying smoothness assumptions [7].

In this paper, we use an idea of [6], where rates of convergence of interpolants were obtained for $S^{2}$ in case the interpolation points corresponded to gridded points on the rectangle of angles for the parameterization of $S^{2}$. However, in this paper, we are able to utilize specific results about spherical harmonics to extend these rates of convergence to the scattered case. The results of Bos et al. [1] on tangential Markov inequalities proved very helpful in this respect.

There are totally different approaches to interpolation and representation of data on $S^{2}$ which mainly use tensor products of polynomial and trigonometric splines on the rectangular parameterization of $S^{2}$. For an account of results in this direction the reader might consult [3], [20].

The outline of the paper is as follows. In Section 2 we collect results about spherical harmonics and the series representation of functions and distributions on the sphere. The tangential Markov inequality for spherical harmonics is also formulated. It is applied in Section 3 in conjunction with the notion of norming sets. We prove the existence of lower bounds for the sampling operator

$$
T(f)=\left.f\right|_{X}
$$

in the maximum norm, where $X$ is a finite knot set and $f$ is taken from the space of spherical harmonics of maximal degree $\ell$. The lower bound depends on the mesh norm of $X$ (Proposition 1). Section 4 gives a brief introduction to strictly positive definite kernels on $S^{n-1}$ and the underlying native spaces $\mathbf{H}$. The main part of the paper, Section 5, starts with the statement of the general error estimate due to Golomb and Weinberger [10]. Our main theorem, Theorem 2, gives an estimate for the error bound in this theory which depends on the mesh norm and the smoothness of the positive definite kernel. Some consequences are given, which include Sobolev type estimates of the interpolation error. We finish our paper by giving two examples for interpolation on $S^{2}$, in Section 6.

\section{Prerequisites}

2.1. Spherical harmonics. As our main analysis tool we use an orthonormal basis of $L_{2}\left(S^{n-1}\right)$ which consists of spherical harmonics. For the reader's convenience, we describe some basic facts here, and refer to the book [14] for a more elaborate description. 
Let $H_{\ell}$ be a homogeneous polynomial in $\mathbb{R}^{n}$ of degree $\ell$ which satisfies $\Delta H_{\ell}=0$, where $\Delta$ is the Laplacian. Then the restriction $Y_{\ell}=\left.H_{\ell}\right|_{S^{n-1}}$ is called a spherical harmonic of order $\ell$. The dimension of the space $V_{\ell}$ of spherical harmonics of order $\ell$ on $S^{n-1}$ will be denoted by $N(n, \ell)$; hence

$$
N(n, 0)=1, \quad \text { and } \quad N(n, \ell)=\frac{2 \ell+n-2}{\ell}\left(\begin{array}{c}
\ell+n-3 \\
\ell-1
\end{array}\right) \quad \text { for } \quad \ell \geq 1,
$$

see $\left[14\right.$, p. 4]. The space $V_{\ell}$ is known as the eigenspace of the Laplace-Beltrami operator on $S^{n-1}$ for the eigenvalue

$$
\lambda_{\ell}=\ell(\ell+n-2), \quad \ell \geq 0 .
$$

Different eigenspaces $V_{\ell}$ and $V_{m}, \ell \neq m$, are orthogonal with respect to the usual inner product,

$$
\langle f, g\rangle=\int_{S^{n-1}} f(\mathbf{p}) g(\mathbf{p}) d S,
$$

with $d S$ the surface element of $S^{n-1}$. The space of spherical harmonics up to order $\ell$ is given by

$$
\widetilde{V}_{\ell}=\sum_{j=0}^{\ell} V_{j}
$$

and has the dimension $\tilde{N}(n, \ell)=N(n+1, \ell)$. Let

$$
\left\{Y_{\ell, k} ; 1 \leq k \leq N(n, \ell)\right\}
$$

be an orthonormal system of eigenfunctions of $V_{\ell}$. Then the famous addition theorem (see $[14$, Thm. 2]) states that

$$
\sum_{k=1}^{N(n, \ell)} Y_{\ell, k}(\mathbf{p}) Y_{\ell, k}(\mathbf{q})=\frac{N(n, \ell)}{\omega_{n-1}} P_{\ell}(n ; \mathbf{p} \cdot \mathbf{q}), \quad \mathbf{p}, \mathbf{q} \in S^{n-1},
$$

where $P_{\ell}(n ; \cdot)$ is the Legendre polynomial of degree $\ell$ in $n$ dimensions, normalized by $P_{\ell}(n ; 1)=1$. (With this normalization it is a multiple of the Gegenbauer polynomial $C_{\ell}^{(n-2) / 2}$.) The constant $\omega_{n-1}$ denotes the surface area of $S^{n-1}$.

2.2. Functional and distributional spaces. The collection of orthonormal functions

$$
\left\{Y_{\ell, k} ; \ell \geq 0,1 \leq k \leq N(n, \ell)\right\}
$$

is complete in $L_{2}\left(S^{n-1}\right)$. With the coefficients

$$
\widehat{f_{\ell, k}}=\int_{S^{n-1}} f(\mathbf{p}) Y_{\ell, k}(\mathbf{p}) d S,
$$

for any function $f \in L_{2}\left(S^{n-1}\right)$ its associated (Fourier) series

$$
f=\sum_{\ell=0}^{\infty} \sum_{k=1}^{N(n, \ell)} \widehat{f}_{\ell, k} Y_{\ell, k}
$$

converges in $L_{2}\left(S^{n-1}\right)$. Such expansions (as in the case of periodic functions) can also be defined in a wider sense, namely for distributions on the sphere. Since $S^{n-1}$ is compact, these distributions are the series (6) with tempered (i.e. polynomially 
bounded) coefficients. The Sobolev space $H^{s}\left(S^{n-1}\right)$ with real parameter $s$ consists of all distributions $f$ such that

$$
\|f\|_{s}^{2}:=\sum_{\ell=0}^{\infty} \sum_{k=1}^{N(n, \ell)}\left(1+\lambda_{\ell}\right)^{s}\left|\widehat{f}_{\ell, k}\right|^{2}<\infty .
$$

For more details we refer to $[11, \S 1.7]$.

2.3. Markov inequality. The restriction of any spherical function $Y_{\ell}$ of order $\ell$ to a great circle (which is a geodesic of the sphere) is a univariate trigonometric polynomial of degree less than or equal to $\ell$. Hence the classical Bernstein inequality $[4$, Section 4.1] implies

$$
\left|D_{T} Y_{\ell}(\mathbf{p})\right| \leq \ell\left\|Y_{\ell}\right\|_{\infty},
$$

where $D_{T}$ denotes any unit tangential derivative at $\mathbf{p}$ and the maximum norm is on $S^{n-1}$. This is a simple case of the general Markov inequality for polynomials on compact smooth algebraic submanifolds of $\mathbb{R}^{n}$ without boundary [1]. We will use it later in its integrated form

$$
|Y(\mathbf{p})-Y(\mathbf{q})| \leq \ell d(\mathbf{p}, \mathbf{q})\|Y\|_{\infty}, \quad \mathbf{p}, \mathbf{q} \in S^{n-1}, \quad Y \in \widetilde{V}_{\ell} .
$$

\section{NORMING SETS IN $C\left(S^{n-1}\right)$}

The following notion from Banach space theory will be very useful.

Definition 1. Let $V$ be a normed linear space with dual $V^{*}$. Given two subspaces $W \subset V$ and $Z \subset V^{*}$, the set $Z$ is called a norming set of $W$ if there exists some $c>0$ so that

$$
\sup _{z \in Z,\|z\|=1}|z(w)| \geq c\|w\| \quad \text { for all } \quad w \in W .
$$

By an application of Markov's inequality, we can find the following result for the normed space $C\left(S^{n-1}\right)$.

Proposition 1. Any knot set $X$ with mesh norm $h(X) \leq 1 /(2 \ell)$ gives rise to a norming set $Z=\operatorname{span}\left\{\delta_{x} ; x \in X\right\}$ of $\widetilde{V}_{\ell}$, with constant $c=1 / 2$. Moreover, there exists a norming set of $\widetilde{V}_{\ell}$ in $C\left(S^{n-1}\right)$ of cardinality $\leq$ const $\cdot \ell^{n-1}$, where the constant is independent of $\ell$.

Proof. It suffices to prove the first part of the proposition, since then the second part follows by standard covering arguments. Hence let us assume that $X$ is given as in the assumption of the theorem, and let $Y \in \widetilde{V}_{\ell},\|Y\|_{\infty}=1$. Then $|Y(\mathbf{p})|=1$ for some $\mathbf{p} \in S^{n-1}$. Hence we can pick $x \in X$ so that $d(\mathbf{p}, x)<(1+\epsilon) /(2 \ell)$, where $\epsilon>0$ can be arbitrarily small. Markov's inequality implies

$$
|Y(\mathbf{p})-Y(x)| \leq \ell d(\mathbf{p}, x)\|Y\|_{\infty}<(1+\epsilon) / 2 .
$$

We thus have shown that $|Y(x)|>(1-\epsilon) / 2$, and letting $\epsilon$ go to zero proves the proposition.

We concentrate on point evaluations as functionals in $V^{*}$ next. Given a finite knot set $X \subset S^{n-1}$, we call

$$
T: C\left(S^{n-1}\right) \rightarrow C(X),\left.\quad f \mapsto f\right|_{X},
$$


the sampling operator. Obviously, $T$ is a contraction. Since $X$ is discrete, $C(X)$ may be identified with $\mathbb{R}^{|X|}$, equipped with the maximum norm. Hence its dual is

$$
(C(X))^{*}=\operatorname{span}\left\{\left.\delta_{x}\right|_{C(X)} ; x \in X\right\},
$$

where $\left.\delta_{x}\right|_{C(X)}: C(X) \rightarrow \mathbb{R}$ denotes the evaluation functional when restricted to $C(X)$ instead of $C\left(S^{n-1}\right)$. The adjoint of the operator $T$ is the isometric imbedding of $(C(X))^{*}$ into $\left(C\left(S^{n-1}\right)\right)^{*}$. These are some preparatory remarks for the following application of norming sets.

Proposition 2. Let $W$ be a finite dimensional subspace of $C\left(S^{n-1}\right)$ and $Z=$ $\operatorname{span}\left\{\delta_{x} ; x \in X\right\}$, where $X$ is a finite knot set. Assume that $Z$ is a norming set of $W$ with norming constant $c \geq 1 / 2$. Then $W^{*}$ can be identified with the space $\operatorname{span}\left\{\left.\delta_{x}\right|_{W} ; x \in X\right\}$. Moreover any $w^{*} \in W^{*},\left\|w^{*}\right\|=1$, can be identified with some

$$
\left.\sum_{x \in X} a_{x} \delta_{x}\right|_{W}, \quad \text { where } \quad \sum_{x \in X}\left|a_{x}\right| \leq 2 .
$$

Proof. We consider the sampling operator restricted to $W$,

$$
T_{0}=\left.T\right|_{W}: W \rightarrow T(W)
$$

Since $Z$ is a norming set of $W, T_{0}$ is a one-to-one isomorphism and $\left\|T_{0}^{-1}\right\| \leq 2$. Also

$$
T_{0}^{*}:(T(W))^{*} \rightarrow W^{*}
$$

is an isomorphism with $\left\|\left(T_{0}^{*}\right)^{-1}\right\| \leq 2$. In other words, for any $w^{*} \in W^{*}$ with $\left\|w^{*}\right\|=1$, there is

$$
t^{*} \in(T(W))^{*} \quad \text { so that } \quad T_{0}^{*}\left(t^{*}\right)=w^{*}, \quad\left\|t^{*}\right\| \leq 2 .
$$

Finally, $T(W)$ is a finite-dimensional subspace of $C(X)$. Thus, by the Hahn-Banach theorem, every $t^{*} \in(T(W))^{*}$ extends to a functional

$$
l^{*} \in(C(X))^{*}, \quad l^{*}=\left.\sum_{x \in X} a_{x} \delta_{x}\right|_{C(X)},
$$

so that

$$
t^{*}=\left.l^{*}\right|_{T(W)} \quad \text { and } \quad\left\|t^{*}\right\|=\left\|l^{*}\right\|=\sum_{x \in X}\left|a_{x}\right| \leq 2 .
$$

For fixed $w^{*} \in W^{*}$ let us denote by $l^{*}=\left.\sum_{x} a_{x} \delta_{x}\right|_{C(X)}$ this extension of the functional $\left(T_{0}^{*}\right)^{-1} w^{*}$. Then obvious transformations give

$$
\begin{aligned}
\left\langle w^{*}, w\right\rangle & =\left\langle w^{*}, T_{0}^{-1} T_{0} w\right\rangle=\left\langle\left(T_{0}^{*}\right)^{-1} w^{*}, T_{0} w\right\rangle \\
& =\left\langle\left.\sum_{x} a_{x} \delta_{x}\right|_{C(X)}, T_{0} w\right\rangle=\left\langle\left.\sum_{x} a_{x} \delta_{x}\right|_{W}, w\right\rangle
\end{aligned}
$$

for all $w \in W$. We thus have completed the proof of Proposition 2.

Remark. The above situation can be compared to usual Lagrange interpolation. If the knot set $X$ from above has the same cardinality as the dimension of $W$, then the operator $T_{0}^{-1}$ is identical to the interpolation operator that maps arbitrary real data on $X$ to an interpolating function in $W$. Hence the lower constant $c$ of the norming set can be chosen as the reciprocal of the so-called Lebesgue constant, which is the norm of the interpolation operator. In this sense, the notion of norming 
sets gives us more freedom, since we can work with a larger knot set $X$. We make use of these degrees of freedom in order to obtain uniform bounds for $c$. On the other hand, there is no known sequence of knot sets for Lagrange interpolation on spheres, which gives rise to a uniform bound of the Lebesgue constants. Best results in this direction may be obtained through interpolation on the basis of extremal fundamental systems as described, e.g., in Reimer's book [17, Chapters 14 and 15].

Let us summarize our findings in the following result which applies to spherical harmonics.

Corollary 1. Let $X \subset S^{n-1}$ be a finite knot set with mesh norm $h(X) \leq 1 /(2 \ell)$. Then for any linear functional $v^{*}$ on $\widetilde{V}_{\ell},\left\|v^{*}\right\|=1$, there exists $\left(a_{x}\right)_{x \in X}$ with $\sum_{x \in X}\left|a_{x}\right| \leq 2$ so that

$$
v^{*}(Y)=\left\langle\sum_{x \in X} a_{x} \delta_{x}, Y\right\rangle, \quad Y \in \widetilde{V}_{\ell} .
$$

Moreover, there exist such knot sets whose cardinality is bounded by a multiple of $\ell^{n-1}$.

\section{Positive definite Kernels and the native space}

In this and the next section we describe the variational approach to scattered data interpolation. The general framework was developed by Golomb and Weinberger in 1959 [10]. We put special emphasis on positive definite kernels in $C\left(S^{n-1} \times S^{n-1}\right)$

$$
\kappa(\mathbf{p}, \mathbf{q})=\sum_{\ell=0}^{\infty} \sum_{k=1}^{N(n, \ell)} a_{\ell, k} Y_{\ell, k}(\mathbf{p}) Y_{\ell, k}(\mathbf{q}), \quad \mathbf{p}, \mathbf{q} \in S^{n-1},
$$

with all coefficients $a_{\ell, k}>0$. We further assume from the beginning that $\kappa \in$ $H^{2 s}\left(S^{n-1} \times S^{n-1}\right)$ for some $s \geq 0$, or equivalently

$$
\sum_{\ell=0}^{\infty}\left(1+2 \lambda_{\ell}\right)^{2 s} \sum_{k=1}^{N(n, \ell)} a_{\ell, k}^{2}<\infty .
$$

Kernels of the form (8) are called convolution kernels, since they act by multiplication in the Fourier domain, e.g.

$$
\int_{S^{n-1}} f(\mathbf{p}) \kappa(\mathbf{p}, \mathbf{q}) d \mathbf{p}=\sum_{\ell, k} a_{\ell, k} \widehat{f}_{\ell, k} Y_{\ell, k}(\mathbf{q}) .
$$

They give rise to generalized Hermite interpolation as studied in the papers [6], [15], which also deal with the unisolvency of these interpolation problems.

The kernel $\kappa$ defines a Hilbert space

$$
\mathbf{H}_{\kappa}:=\left\{f \in D^{\prime}\left(S^{n-1}\right) ;\|f\|_{\kappa}^{2}=\sum_{\ell, k} \frac{\left|\widehat{f}_{\ell k}\right|^{2}}{a_{\ell, k}}<\infty\right\},
$$

the so-called native space for interpolation. This notion was introduced by Madych and Nelson [12] for the study of scattered data interpolation in Euclidean space $\mathbb{R}^{n}$. Here we are in a different situation since $S^{n-1}$ is compact and has a discrete Fourier domain. Note that our assumptions on $\kappa$ yield $\mathbf{H}_{\kappa} \subset H^{s}\left(S^{n-1}\right)$ (which is a continuous imbedding), since $a_{\ell, k}=o\left(1+2 \lambda_{\ell}\right)^{-s}$ by $(9)$. Hence the Lagrange 
or Hermite interpolation problems can be posed as long as the Sobolev Imbedding Theorem guarantees that these functionals are continuous on $\mathbf{H}_{\kappa}$. In some cases of interest the native space is even contained in $C^{\infty}\left(S^{n-1}\right)$. This follows if the kernel has exponentially decaying $a_{\ell, k}$, which occurs for generalizations of Gaussian or multiquadric kernels of the Euclidean space.

\section{ERror estimates For Lagrange interpolation}

5.1. General interpolation. Our error estimates are given in terms of Fourier expansions (6). The main idea is taken from the paper [6]. We give a somewhat different description here, which we think is more closely related to the classical form of interpolation as treated by Golomb and Weinberger [10]. It also avoids the restriction to a subspace of the native space $\mathbf{H}_{\kappa}$, which intrinsically appears in [6].

Theorem 1 [10]. Let $L_{m}, 1 \leq m \leq M$, be linearly independent continuous linear functionals on the native space $\mathbf{H}_{\kappa}$. We define the space

$$
\begin{aligned}
& \qquad \begin{array}{r}
F=\left\{v \in \mathbf{H}_{\kappa} ; L_{m}(v)=0 \text { for all } 1 \leq m \leq M\right\}, \\
\text { and for given data } d=\left(d_{m} ; 1 \leq m \leq M\right) \in \mathbb{R}^{M} \text { let } \\
F_{d}=\left\{v \in \mathbf{H}_{\kappa} ; L_{m}(v)=d_{m} \text { for all } 1 \leq m \leq M\right\} .
\end{array}
\end{aligned}
$$

Then there exists a unique interpolant $u \in F^{\perp} \cap F_{d}$; i.e.

$$
L_{m}(u)=d_{m} \quad \text { for all } \quad 1 \leq m \leq M .
$$

This interpolant satisfies the following properties:

(M1) First minimum property: For any $v \in F_{d}$

$$
\|v\|_{\kappa}^{2}=\|u\|_{\kappa}^{2}+\|v-u\|_{\kappa}^{2} .
$$

(M2) Second minimum property: For another continuous linear functional $L$ on $\mathbf{H}_{\kappa}$ the value $L u$ is the best approximation to $\left\{L v ; v \in F_{d},\|v\|_{\kappa}=r\right\}$, meaning that

$$
\sup _{v \in C_{r}}|L v-L w| \geq \sup _{v \in C_{r}}|L v-L u|
$$

holds for all $w \in \mathbf{H}_{\kappa}$. Here $C_{r}:=\left\{v \in F_{d} ;\|v\|_{\kappa}=r\right\}, r>0$, denotes a hypercircle, and if $C_{r}$ is non-empty, then equality holds only for $w=u$.

Furthermore, the hypercircle inequality is satisfied: If $y \in F$ denotes an element with unit norm for which $\left.L\right|_{F}$ attains its least upper bound, then

$$
|L v-L u|^{2} \leq|L y|^{2}\left(r^{2}-\|u\|_{\kappa}^{2}\right) \quad \text { for all } \quad v \in C_{r} .
$$

Let us add a few remarks to this theorem. The space $F^{\perp}$ is often called a spline space. It is spanned by the basis functions

$$
u_{m}(\mathbf{p}):=L_{m} \kappa(\cdot, \mathbf{p}), \quad 1 \leq m \leq M .
$$

Secondly, the term $|L y|$ in the hypercircle inequality can be written as

$$
|L y|=\operatorname{dist}_{\kappa}\left(v_{L}, F^{\perp}\right),
$$

where $v_{L}$ is the representer of the linear functional $L$ in the native space and the distance is taken in the norm of this space. In this form the hypercircle inequality was given in [6]. The term $|L y|$, for special choice of the functional $L$, is sometimes called the power function (Schaback [18]). 
Our aim is to give estimates for $|L(y)|$ in the hypercircle inequality. This is done by eliminating a number of Fourier coefficients and finding bounds on the 'tails' by means of appropriate assumptions on the kernel $\kappa$. If we employ the expansion (6) of $y$, then we obtain by orthogonality

$$
|L y|=\left|\left(L-\sum_{m=1}^{M} c_{m} L_{m}\right) y\right| \leq \sum_{\ell=0}^{\infty} \sum_{k=1}^{N(n, \ell)}\left|\left(L-\sum_{m=1}^{M} c_{m} L_{m}\right) Y_{\ell, k}\right|\left|\widehat{y}_{\ell, k}\right|
$$

for arbitrary coefficients $c_{m} \in \mathbb{R}$. Let us assume for the moment that the $c_{m}$ can be chosen in such a way that

$$
\left(L-\sum_{m=1}^{M} c_{m} L_{m}\right) Y_{\ell, k}=0 \quad \text { for all } \quad \ell \leq \Lambda, 1 \leq k \leq N(n, \ell) .
$$

Then the Cauchy-Schwarz inequality gives

$$
\begin{aligned}
|L y|^{2} & \leq \sum_{\ell>\Lambda} \sum_{k=1}^{N(n, \ell)} \frac{\left|\widehat{y}_{\ell, k}\right|^{2}}{a_{\ell, k}} \cdot \sum_{\ell>\Lambda} \sum_{k=1}^{N(n, \ell)} a_{\ell, k}\left|\left(L-\sum_{m=1}^{M} c_{m} L_{m}\right) Y_{\ell, k}\right|^{2} \\
& \leq \sum_{\ell>\Lambda} \sum_{k=1}^{N(n, \ell)} a_{\ell, k}\left|\left(L-\sum_{m=1}^{M} c_{m} L_{m}\right) Y_{\ell, k}\right|^{2}, \quad \text { since }\|y\|_{\kappa}=1 .
\end{aligned}
$$

This is a reformulation of Proposition 3.6 in [6], but the restriction to a subspace of $\mathbf{H}_{\kappa}$ was removed. The further technique consists of estimates for the last series, which are not based on the norm of the native space, but on some easily accessible norm (like $\|\cdot\|_{\infty^{-}}$or Sobolev norm).

5.2. Lagrange interpolation by splines. In this paper we concentrate on error estimates for Lagrange interpolation. The native space setting is as follows. We are given a set of interpolation knots

$$
X=\left\{x_{m} ; 1 \leq m \leq M\right\} \subset S^{n-1}
$$

whose mesh norm is

$$
h(X):=\sup _{\mathbf{p} \in S^{n-1}} d(\mathbf{p}, X),
$$

see (1). The functionals to be considered in Theorem 5.1 are $L_{m}=\delta_{x_{m}}, 1 \leq m \leq$ $M$. The pointwise (or $L_{\infty^{-}}$) error of interpolation is measured by

$$
L=\delta_{\mathbf{p}} \quad \text { with } \quad \mathbf{p} \in S^{n-1} \text { fixed, }
$$

which represents the functional to be approximated. The 'splines' in the interpolation space $F^{\perp}$ are defined by

$$
u_{m}(\mathbf{p})=L_{m} \kappa(\cdot, \mathbf{p})=\kappa\left(x_{m}, \mathbf{p}\right), \quad 1 \leq m \leq M .
$$

Let us also use the notation

$$
\widehat{a}_{\ell}:=\max _{1 \leq k \leq N(n, \ell)} a_{\ell, k} \quad \text { and } \quad x_{0}=\mathbf{p} .
$$

We can now formulate the main result of this paper. It makes use of Corollary 1 in order to give estimates for the pointwise interpolation error. 
Theorem 2. Assume that the set of interpolation knots $X \subset S^{n-1}$ has mesh norm $h(X) \leq 1 /(2 \Lambda)$, for some $\Lambda \in \mathbb{N}$. Then there exist numbers $c_{m} \in \mathbb{R}, 1 \leq m \leq M$, so that

$$
\left(L-\sum_{m=1}^{M} c_{m} L_{m}\right) Y=0 \quad \text { for all } Y \in \widetilde{V}_{\Lambda}, \quad \text { and } \quad \sum_{m}\left|c_{m}\right| \leq 2 .
$$

Moreover, the term $|L y|^{2}$ in the hypercircle inequality (11) can be bounded by

$$
\begin{aligned}
|L y|^{2} & \leq \frac{5}{\omega_{n-1}} \cdot \sum_{\ell>\Lambda} \widehat{a}_{\ell} N(n, \ell) \lambda_{\max }\left(P_{\ell}\left(n ; x_{\mu} \cdot x_{\nu}\right)\right)_{0 \leq \mu, \nu \leq M} \\
& \leq \frac{5(M+1)}{\omega_{n-1}} \sum_{\ell>\Lambda} \widehat{a}_{\ell} N(n, \ell) .
\end{aligned}
$$

Here $P_{\ell}(n ; \cdot)$ is the Legendre polynomial in $n$ dimensions, and $\lambda_{\max }$ denotes the maximal eigenvalue of the symmetric, positive semi-definite matrix.

Proof. The existence of the coefficients $\left(c_{m}\right)$ with $\sum_{m}\left|c_{m}\right| \leq 2$ is a direct consequence of Corollary 3.3. Next we give bounds for the last series in (5.4). Let $x_{0}=\mathbf{p}, c_{0}=-1$. If we use the Addition Theorem (4) in the second line, then the inner series in (13) gives

$$
\begin{aligned}
\sum_{k=1}^{N(n, \ell)} a_{\ell, k}\left|\left(L-\sum_{m=1}^{M} c_{m} L_{m}\right) Y_{\ell, k}\right|^{2} & =\sum_{k=1}^{N(n, \ell)} a_{\ell, k}\left|\sum_{m=0}^{M} c_{m} Y_{\ell, k}\left(x_{m}\right)\right|^{2} \\
& \leq \widehat{a}_{\ell} \cdot \sum_{k=1}^{N(n, \ell)} \sum_{\mu, \nu=0}^{M} c_{\mu} c_{\nu} Y_{\ell, k}\left(x_{\mu}\right) Y_{\ell, k}\left(x_{\nu}\right) \\
& =\widehat{a}_{\ell} \cdot \frac{N(n, \ell)}{\omega_{n-1}} \sum_{\mu, \nu=0}^{M} c_{\mu} c_{\nu} P_{\ell}\left(n ; x_{\mu} \cdot x_{\nu}\right)
\end{aligned}
$$

The matrix $\left(P_{\ell}\left(n ; x_{\mu} \cdot x_{\nu}\right)\right)_{\mu, \nu}$ is positive semi-definite and symmetric. Hence we proved the first estimate in the theorem, since

$$
\sum_{m=0}^{M}\left|c_{m}\right|^{2} \leq\left|c_{0}\right|^{2}+\left(\sum_{m=1}^{M}\left|c_{m}\right|\right)^{2} \leq 5
$$

The second inequality follows from the fact that each entry in the above matrix has absolute value $\leq 1$ (cf. [14, Lemma 9]) and Gershgorin's circle theorem. Thus the proof of Theorem 2 is completed.

Let us note that the estimate of the maximal eigenvalue of the given matrix $\left(P_{\ell}\left(n ; x_{\mu} \cdot x_{\nu}\right)\right)_{\mu, \nu}$ is rather pessimistic. There are a few known configurations of knots where $\lambda_{\max }=1$, cf. [17, Chapter 13]. This is also the least possible value, since the trace of the matrix is given by

$$
\sum_{m=0}^{M} P_{\ell}(n ; 1)=M+1
$$

Further investigations in order to improve the estimate of $\lambda_{\max }$ are deferred to future research. 
Many interesting kernels $\kappa$ as in (8) have the form

$$
\kappa(\mathbf{p}, \mathbf{q})=f(\mathbf{p} \cdot \mathbf{q})=\omega_{n-1}^{-1} \sum_{\ell=0}^{\infty} \widehat{a}_{\ell} N(n, \ell) P_{\ell}(n ; \mathbf{p} \cdot \mathbf{q}),
$$

where $f$ is a univariate function in $C^{k}[-1,+1]$. Hence the Fourier coefficients in (8) are $a_{\ell, k}=\widehat{a}_{\ell}$, by means of the Addition Theorem (4). For these kernels the Sobolev estimate (9) takes the form

$$
\kappa \in H^{2 s}\left(S^{n-1} \times S^{n-1}\right) \Longleftrightarrow \sum_{\ell=0}^{\infty}(1+2 \ell(\ell+n-2))^{2 s} \widehat{a}_{\ell} N(n, \ell)<\infty .
$$

This implies that

$$
\widehat{a}_{\ell} N(n, \ell)=o\left(\ell^{-4 s}\right) \quad \text { for } \quad \ell \rightarrow \infty .
$$

In our subsequent error estimates, which are simple consequences of Theorem 2 , we will therefore use assumptions on the decay of the sequence $\left(\widehat{a}_{\ell} N(n, \ell)\right)_{\ell \geq 0}$, where $\widehat{a}_{\ell}$ is defined in (14).

Corollary 2. Let $\kappa$ be a positive definite kernel in $C\left(S^{n-1} \times S^{n-1}\right)$ as in (8) with all Fourier coefficients $a_{\ell, k}>0$. Furthermore, let $X=\left\{x_{1}, \ldots, x_{M}\right\} \subset S^{n-1}$ be a knot set with mesh norm $h=h(X)$, and let $0<\Lambda \in \mathbb{N}$ be such that $1 /(2 \Lambda+2)<$ $h(X) \leq 1 /(2 \Lambda)$.

Then for given $f \in \mathbf{H}_{\kappa}$, there is a unique interpolant

$$
u \in \operatorname{span}\{\kappa(x, \cdot) ; x \in X\}
$$

with $\left.f\right|_{X}=\left.u\right|_{X}$ and satisfying the error estimate

$$
\|f-u\|_{\infty}^{2} \leq \frac{5(M+1)}{\omega_{n-1}}\|f\|_{\kappa}^{2} \sum_{\ell>\Lambda} \widehat{a}_{\ell} N(n, \ell), \quad \widehat{a}_{\ell}=\max _{1 \leq k \leq N(n, \ell)} a_{\ell, k} .
$$

Moreover, with special assumptions on the decay of the coefficients $\widehat{a}_{\ell}$ we have approximation orders as follows:

- If $\widehat{a}_{\ell} N(n, \ell) \leq c_{1}(1+\ell)^{-\alpha}$ for some $\alpha>1$, then

$$
\begin{aligned}
\|f-u\|_{\infty}^{2} & \leq \frac{5 c_{1}}{\omega_{n-1}}\|f\|_{\kappa}^{2}(M+1) \int_{\Lambda}^{\infty} \frac{d \ell}{(1+\ell)^{\alpha}} \\
& =\frac{5 c_{1}}{(\alpha-1) \omega_{n-1}}\|f\|_{\kappa}^{2}(M+1)(1+\Lambda)^{1-\alpha} .
\end{aligned}
$$

- If $\widehat{a}_{\ell} N(n, \ell) \leq c_{1} e^{-\alpha(1+\ell)}$ for some $\alpha>0$, then

$$
\begin{aligned}
\|f-u\|_{\infty}^{2} & \leq \frac{5 c_{1}}{\omega_{n-1}}\|f\|_{\kappa}^{2}(M+1) \int_{\Lambda}^{\infty} e^{-\alpha(1+\ell)} d \ell \\
& =\frac{5 c_{1}}{\alpha \omega_{n-1}}\|f\|_{\kappa}^{2}(M+1) e^{-\alpha(1+\Lambda)} .
\end{aligned}
$$

It should be noted that the factor $M+1$ will usually depend on $h(X)$ as well. A natural assumption would be (see Proposition 1) that

$$
h(X)^{n-1}(M+1) \leq \text { const } .
$$

We can thus give approximation orders in terms of the mesh norm of $X$.

Corollary 3. Let $X$ be any knot set on $S^{n-1}$ whose mesh norm $h$ satisfies (16). 
(a) If $\widehat{a}_{\ell} N(n, \ell) \leq c_{1}(1+\ell)^{-\alpha}$ for some $\alpha>n$, then

$$
\|f-u\|_{\infty}^{2}=\mathbf{O}\left(h^{\alpha-n}\right) .
$$

(b) If $\widehat{a}_{\ell} N(n, \ell) \leq c_{1} e^{-\alpha(1+\ell)}$ for some $\alpha>0$, then

$$
\|f-u\|_{\infty}^{2}=\mathbf{O}\left(h^{1-n} e^{-\alpha / 2 h}\right) .
$$

The constants in these upper bounds can be chosen to depend only on $c_{1}, \alpha$, the constant in (16), the dimension $n$ and the norm $\|f\|_{\kappa}$.

The upper bound in (b) is sometimes called 'spectral approximation order', since the convergence as $h$ tends to 0 is faster than any polynomial in $h$.

\section{Two FAMILIES OF KERNELS AND NUMERICAL RESUlTS}

In this last section we present numerical experiments for scattered data interpolation on $S^{2}$. Let us begin with the discussion of two different families of kernels on $S^{n-1}$ which have the special form (15). The first kind consists of $C^{\infty}$-functions with exponentially decaying Fourier coefficients; hence part (b) in Corollary 3 applies to this family. They are defined by a generating function of the Legendre polynomials. From [14, p. 30], we have that, for $0<z<1$,

$$
\kappa_{z}^{\mathrm{gen}}(\mathbf{p}, \mathbf{q})=\frac{1-z^{2}}{\left(1+z^{2}-2 z \mathbf{p} \cdot \mathbf{q}\right)^{n / 2}}=\sum_{\ell=0}^{\infty} N(n, \ell) z^{\ell} P_{\ell}(n ; \mathbf{p} \cdot \mathbf{q}) .
$$

The exponential decay and positivity of the Fourier coefficients is obvious from (19).

The second family is defined as follows. For any even $\nu \in \mathbb{N}$ we let

$$
\kappa_{\nu}(\mathbf{p}, \mathbf{q})=\sum_{\ell=0}^{\infty} N(n, \ell) a_{\ell}^{(\nu)} P_{\ell}(n ; \mathbf{p} \cdot \mathbf{q})=c_{0}+\sum_{k=1}^{\infty} \frac{(\mathbf{p} \cdot \mathbf{q})^{k}}{k^{\nu}}, \quad c_{0}>0 .
$$

This is a continuous function, since the power series $\sum_{k} z^{k} / k^{\nu}$ converges uniformly in the interval $[-1,+1]$ due to our assumption $\nu>1$. Strict positivity of the coefficients in this power series also implies that all coefficients $a_{\ell}^{(\nu)}$ in the Fourier expansion are positive. This very general fact is a consequence of Schoenberg's work [19] on positive definite functions on spheres. The power series are sometimes called poly-logarithmic functions and are discussed in connection with the zetafunction. MATHEMATICA has an intrinsic definition for them. By some more detailed analysis one can show that

$$
N(n, \ell) a_{\ell}^{(\nu)}=O\left(\ell^{1-2 \nu}\right) \quad \text { for } \quad \ell \rightarrow \infty,
$$

and this order cannot be improved. In particular, the decay rate is independent of the dimension $n$. Hence these kernels provide examples for the assumptions of Corollary $3(\mathrm{a})$.

We use three different knot sets of cardinalities 20, 100 and 400 on $S^{2}$ for our computations. They are 'almost' equidistributed on the sphere and were computed by software which was kindly given to us by U. Depczynski. The mesh norms were computed numerically. We found values of $0.66,0.29$ and 0.17 for the corresponding mesh norms. Similar knot configurations were also developed by J. Fliege and U. Maier [13]. Figure 1 shows the knot locations as seen from one side of the sphere for the two larger knot sets. 

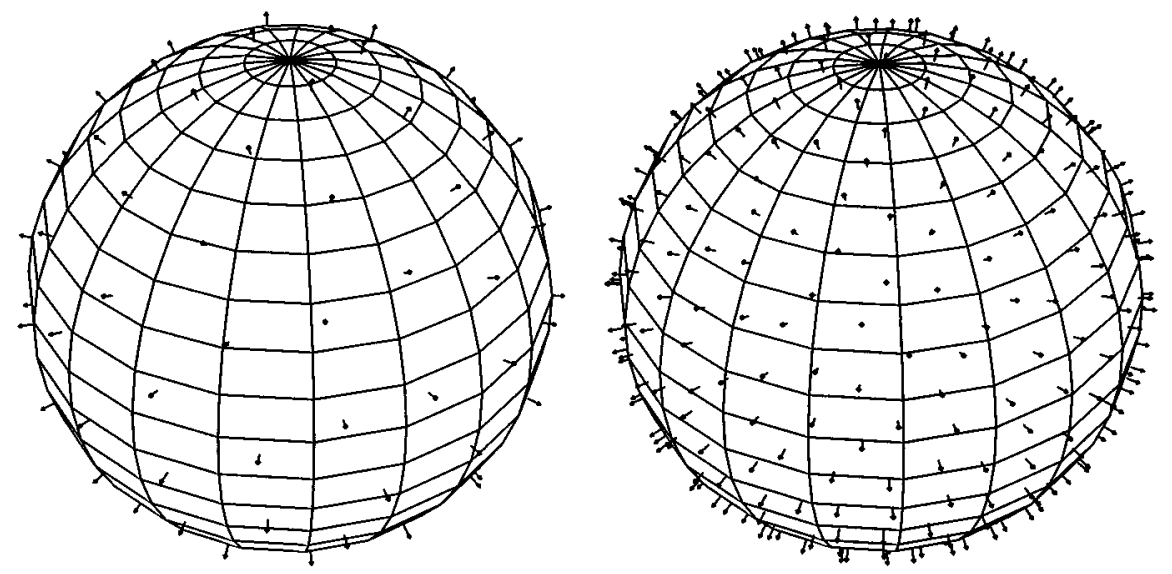

FigurE 1. Spheres with 100 and 400 knots, denoted by small arrows

TABLE 1. Parameters for the definition of the function $f$

\begin{tabular}{|c|cccccc}
$\mathrm{i}$ & $\mathbf{p}_{i, 1}$ & $\mathbf{p}_{i, 2}$ & $\mathbf{p}_{i, 3}$ & $n_{i}$ & $\alpha_{i}$ & $c_{i}$ \\
\hline 1 & 0 & 0 & 1 & 1 & 5 & 2 \\
2 & 0.932039 & 0 & 0.362358 & 1 & 7 & 0.5 \\
3 & -0.362154 & 0.6 .1228 & 0.696707 & 2 & 6 & -2 \\
4 & 0.904035 & 0.279651 & -0.32329 & 1 & 5 & -2 \\
5 & -0.0479317 & -0.424684 & -0.904072 & 1 & 2.1 & 0.2
\end{tabular}

The function $f$ to be interpolated is synthesized by taking the sum of 5 exponentials:

$$
f_{i}(\mathbf{q})=c_{i} e^{-\alpha_{i}\left(1-\mathbf{p}_{i} \cdot \mathbf{q}\right)^{n_{i}}}, \quad 1 \leq i \leq 5, \quad \mathbf{q} \in S^{2}
$$

with some rather arbitrary choices for the parameters $c_{i}, \alpha_{i}, n_{i}$ and the 'centers' $\mathbf{p}_{i} \in S^{2}$ which are given in Table 1 . Each $f_{i}$ is certainly in $C^{\infty}$, and it is closely related to a Gaussian centered at $\mathbf{p}_{i}$ and involving the $\left(2 n_{i}\right)$-th power of the geodesic distance from this point. Hence $f$ is an element of a large variety of native spaces. We depict a surface plot of $(3+f(\mathbf{p})) \mathbf{p}$ in Figure 2 in order to give a better picture of the range of the function values of $f$, which is the interval from -1.85 to 1.28 .

We then compute three different interpolants to the scattered data which is defined by the function values of $f$ on one of the knot sets. Our first interpolant is based on the kernel of type (20) with least possible parameter $\nu=2$. The second interpolant is of the same type, but uses this kernel with parameter $\nu=4$. Finally, the third interpolant is based on the kernel $\kappa_{0.5}^{\text {gen }}$ in (19); hence the parameter $z=1 / 2$ is used here. The maximal deviation $\|f-u\|_{\infty}$, where $u$ is the interpolant, is computed on a rectangular grid of angles, which is of size $100 \times 100$. These maximal errors and sharp numerical estimates for the mesh norm of $X$ are included in Table 2. Our computations of interpolants and mesh norms were done with Matlab 5 on a Pentium 166 MMX. The knot generating software is a $\mathrm{C}++$-routine, which also runs on the same machine. All examples required at most 2 minutes for the knot generation and 3 minutes for the Matlab routines for interpolation and error estimates. 


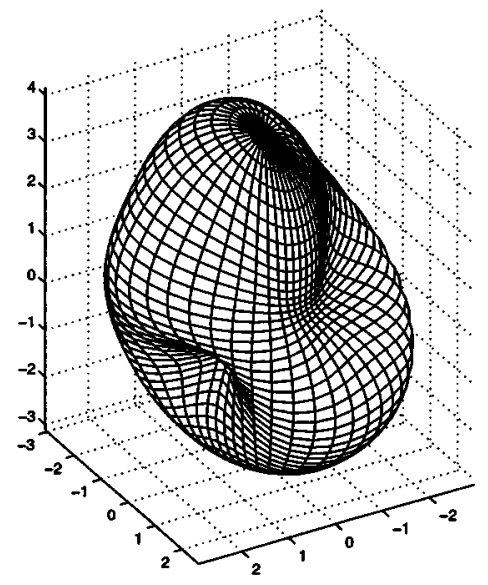

Figure 2. Surface plot of $(3+f(\mathbf{p})) * \mathbf{p}$, where $f$ is the superposition of five Gaussians on the sphere

TABLE 2. $L_{\infty}$-errors for interpolation with different kernels

\begin{tabular}{c|cccc} 
& & \multicolumn{4}{c}{ \# of points in $X$} \\
& & 20 & 100 & 400 \\
\hline mesh norm & $h(X)$ & 0.66 & 0.29 & 0.17 \\
\hline \multirow{3}{*}{$L_{\infty}$-errors with kernel } & $\kappa_{2}$ & 0.700 & 0.060 & 0.0073 \\
& $\kappa_{4}$ & 0.552 & 0.017 & 0.00046 \\
& $\kappa_{0.5}^{\text {gen }}$ & 0.716 & 0.019 & 0.000077 \\
\hline
\end{tabular}

Our numerical data allow a first observation to be made. There is a nice coincidence of the theoretical results in Section 5 and the numerical result in Table 2 . As it should be expected from the smoothness properties of $f$, the smoother interpolants give lower error bounds for the interpolation. We defer more extensive numerical testing and more precise asymptotic error estimates for the specified kernels in (19), (20) to the future.

Let us end our presentation with a remark. The interpolating spline spaces which are studied in this paper have a natural meaning for multiresolution analysis on the sphere. Nested subspaces of the native space are simply obtained by nested knot sequences. First results in this direction were recently obtained in [9], [16].

\section{ACKNowledgments}

We are grateful to L. Bos who pointed us to the fact that Bernstein's inequality can be applied to tangential derivatives of spherical harmonics, and to W. B. Johnson for helpful discussions. We also thank U. Depczynski for making his software available to us. 


\section{REFERENCES}

[1] L. Bos, N. Levenberg, P. Milman, and B. A. Taylor, Tangential Markov inequalities characterize algebraic submanifolds of $R^{N}$, Indiana Univ. Math. J. 44 (1995), 115-138. MR 96i: 41009

[2] E. W. Cheney, Approximation using positive definite functions, Approximation Theory VIII, vol. 1: Approximation and Interpolation (C. K. Chui and L. L. Schumaker, eds.), World Scientific, Singapore, 1995, pp. 145-168. MR 98e:41036

[3] S. Dahlke, W. Dahmen, E. Schmitt and I. Weinreich, Multiresolution analysis and wavelets on $S^{2}$ and $S^{3}$, Numer. Funct. Anal. Optim. 16 (1995), 19-41. MR 96a:42044

[4] R. A. DeVore and G. G. Lorentz, Constructive Approximation, Springer-Verlag, BerlinHeidelberg, 1993. MR 95f:41001

[5] J. Duchon, Splines minimizing rotation-invariant semi-norms in Sobolev spaces, Constructive Theory of Functions of Several Variables (W. Schempp and K. Zeller, eds.), Springer-Verlag, Berlin-Heidelberg, 1979, pp. 85-100. MR 58:12146

[6] N. Dyn, F. J. Narcowich, and J. D. Ward, Variational principles and Sobolev-type estimates for generalized interpolation on a Riemannian manifold, Constr. Approx. (to appear).

[7] W. Freeden, On spherical spline interpolation and approximation, Math. Methods Appl. Sci. 3 (1981), 551-575. MR 83m:41003

[8] W. Freeden and E. W. Grafarend, Mathematische Methoden der Geodäsie, Oberwolfach Conference Report 41/1995.

[9] W. Freeden and U. Windheuser, Combined spherical harmonic and wavelet expansion - A future concept in earth's gravitational determination, Appl. Comput. Harmon. Anal. 4 (1997), 1-37. MR 97j:86004

[10] M. Golomb and H. F. Weinberger, Optimal approximation and error bounds, On Numerical Approximation (R. E. Langer, ed.), The Univ. of Wisconsin Press, Madison, 1959, pp. 117190. MR 22:12697

[11] J. L. Lions and E. Magenes, Non-Homogeneous Boundary Value Problems and Applications, vol. I, Springer-Verlag, New York, 1972. MR 50:2670

[12] W. R. Madych and S. A. Nelson, Multivariate interpolation: a variational theory, manuscript, 1983.

[13] U. Maier and J. Fliege, Charge distribution of points on the sphere and corresponding cubature formulae, Multivariate Approximation: Recent Trends and Results (W. Haußmann, K. Jetter and M. Reimer, eds.), Mathematical Research, vol. 101, Akademie-Verlag, Berlin, 1997, pp. 147-159. CMP 98:12

[14] C. Müller, Spherical Harmonics, Lecture Notes in Mathematics, vol. 17, Springer-Verlag, Berlin-Heidelberg, 1966. MR 33:7593

[15] F. J. Narcowich, Generalized Hermite interpolation and positive definite kernels on a Riemannian manifold, J. Math. Anal. Appl. 190 (1995), 165-193. MR 96c:41009

[16] F. J. Narcowich and J. D. Ward, Nonstationary wavelets on the $m$-sphere for scattered data, Appl. Comput. Harmon. Anal. 3 (1996), 324-336. MR 97h:42020

[17] M. Reimer, Constructive Theory of Multivariate Functions, B. I. Wissenschaftsverlag, Mannheim, 1990. MR 92m:41003

[18] R. Schaback, Comparison of radial basis function interpolants, Multivariate Approximation: From Theory to Software (K. Jetter and F. I. Utreras, eds.), World Scientific, Singapore, 1995, pp. 293-305. MR 96f:65004

[19] I. J. Schoenberg, Positive definite functions on spheres, Duke Math. J. 9 (1942), 96-108. MR 3:232c

[20] L. L. Schumaker and C. Traas, Fitting scattered data on spherelike surfaces using tensor products of trigonometric and polynomial splines, Numer. Math. 60 (1991), 133-144. MR 92j:65012

[21] G. Wahba, Spline interpolation and smoothing on the sphere, SIAM J. Sci. Statist. Comput. 2 (1981), 5-16; errata, ibid. 3 (1982), 385-386. MR 84j:65016a,b

[22] G. Wahba, Surface fitting with scattered noisy data on Euclidian d-space and on the sphere, Rocky Mountain J. Math. 14 (1984), 281-299. MR 86c:65017 
Institut Für Angewandte Mathematik und Statistik, Universität Hohenheim, D-70593 StUtTGART

E-mail address: kjetter@uni-hohenheim.de

InStitut Für Angewandte Mathematik und Statistik, Universität Hohenheim, D-70593 STUtTGART

E-mail address: stockler@uni-hohenheim.de

Department of Mathematics, Texas A\&M University, College Station, TX 77843

E-mail address: jward@math.tamu.edu 